\title{
Competencia económica en el sector de transporte aéreo de pasajeros en México
}

Economic competition in the sector of Air Transport of passengers in Mexico

\author{
Carlos Enrique Cardoso-Vargas*
}

\section{Resumen}

En este trabajo se presenta una revisión sucinta de las condiciones actuales de la competencia económica en el sector de transporte aéreo de pasajeros en México. Además, muestra lo más relevante del marco legal del sector y los eventos más sobresalientes de los últimos años; asimismo, se destacan las condiciones de competencia en el mercado mediante el análisis de índices de concentración, precios, relación entre precios y poder de mercado, competencia intra e inter aeropuertos y las barreras que limitan la libre concurrencia. Los resultados arrojan un ligero incremento en la concentración económica en el sector, sin embargo, en términos de la legislación vigente esta situación no brinda indicios de que el proceso de competencia este siendo afectado. Las principales limitantes en la competencia parecen ser las barreras físicas prevalecientes en la operación del Aeropuerto Internacional de la Ciudad de México y en algunos aspectos de la legislación actual.

\section{Palabras clave:}

- Competencia económica

- Política de competencia

- Sector aéreo en México

\begin{abstract}
This paper presents a brief review of the current conditions of economic competition in the sector of air travel in Mexico. So, this shows the most relevant legal framework for the sector and highlights of recent years; the document also shows the conditions of competition in the market through the analysis of concentration indices, prices, relationship between prices and market power, competition intra and inters airports and barriers to free competition. The results show a slight increase in economic concentration in the sector, however, in terms of the legislation this situation does not provide evidence that this competition process being affected. The main constraints on competition appear to be the prevailing physical barriers in the operation of the Mexico City International Airport and some aspects of current legislation.
\end{abstract}

\section{Keywords: \\ - Economic Competition \\ - Antitrust Policy \\ - Air Transportation}

JEL: D40, L40, L93

\section{Introducción}

En las últimas décadas, a nivel internacional, el sector aerocomercial ha experimentado una serie de cambios como resultado de los procesos de apertura y desregulación implementados en los distintos países. Estados Unidos de América fue el primer país que desreguló este sector a partir del Airline Deregulation Act promulgado en 1978, que redujo las restricciones de entrada al mercado a la vez que eliminó algunas de las limitaciones existentes para la

* Director de Política Fiscal en la Secretaría de Finanzas de la Ciudad de México. Los puntos de vista y conclusiones presentadas en el documento son de exclusiva responsabilidad del autor y no reflejan necesariamente los de la Secretaría de Finanzas de la Ciudad de México. 
fijación de tarifas por parte de las empresas. Como resultado de esta política, el efecto inmediato que se observó fue una reducción de los precios y la entrada de diversas empresas al mercado.

En México, las principales compañías de transporte aéreo se encontraron, en diversos momentos del tiempo, bajo control estatal, no obstante se puede considerar que en 1995 con la publicación de la nueva Ley de Aviación Civil y la Ley de Aeropuertos se sentaron las bases del proceso de desregulación del sector para permitir el ingreso de otras empresas privadas dentro de la industria del transporte aéreo.

En la literatura referente a los aspectos de competencia económica, existen diversos estudios (Ros, 2010; Valdés y Ramírez, 2011; OECD, 2014) que documentan dicho proceso y en algunos casos realizan una evaluación de sus efectos en el sector. Sin embargo, la cobertura temporal de los mismos no permite conocer la situación de la estructura actual y las condiciones de competencia recientes, por lo que este manuscrito busca contribuir abordando esos aspectos faltantes y aportando recomendaciones de política económica sobre los problemas que limitan la libre concurrencia en el sector.

El presente documento se encuentra organizado de la siguiente manera: en la sección II se describe la evolución del proceso de desregulación en la industria aérea en México. La sección III presenta el marco regulatorio vigente; en el apartado IV se examina el estado que guardan las condiciones de competencia y, finalmente, en la última sección se exponen las conclusiones y recomendaciones.

\section{Evolución de la industria de transporte aéreo}

Hasta mediados de los 80's la industria aérea en México se encontraba bajo el control del gobierno federal y existían dos principales empresas estatales, Aeroméxico y Mexicana de Aviación (Mexicana), que concentraban casi en su totalidad el transporte aéreo de pasajeros. En ese tiempo el intervencionismo estatal, derivó en una baja eficiencia operativa y en la aparición de monopolios legales por ruta (Valdés y Ramírez, 2011). La entrada al sector estaba sujeta a diversas concesiones y permisos en donde el proceso de otorgamiento por parte del estado no era transparente ni previsible (Ros, 2010), además la entrega de subsidios y transferencias gubernamentales hacia Aeroméxico y Mexicana tornaban difícil la competencia contra esos dos operadores.

Para revertir esta situación y aumentar la competencia, así como la eficiencia de la aviación comercial en el país, a finales de esa década el gobier- 
no decidió eliminar distintas barreras legales para que compañías distintas al duopolio pudieran servir en rutas regionales y realiza la privatización de Aeroméxico y Mexicana. A principios de los años 90’s, el gobierno permitió la incorporación de nuevos participantes dentro de la industria del transporte aéreo, ${ }^{1}$ con lo cual para 1992 se contaba con más compañías, sirviendo al mercado nacional.

Para 1994 Aeroméxico y Mexicana experimentaron dificultades financieras críticas, ocasionadas por una mala administración e ineficiencias internas, que las llevaron a un colapso económico. Con la finalidad de proveer a estas aerolíneas de la oportunidad de reestructurarse financieramente y evitar la bancarrota, diversos accionistas de la compañía, principalmente bancos mexicanos, constituyeron la Corporación Nacional de Transportación Aérea (Cintra) en los primeros meses de 1995. En ese tiempo, la recién creada Comisión Federal de Competencia (CFC) aprobó la creación de Cintra con la condición que fuera una medida temporal y que eventualmente las aerolíneas serían vendidas en operaciones individuales. ${ }^{2}$

Para finales de 1995, la crisis económica obligó al gobierno federal nuevamente a tomar el mando del duopolio aéreo mediante el control mayoritario de la operación de Cintra, situación que prevaleció hasta la privatización de Mexicana en 2005 y de Aeroméxico en 2007. Para ese año las dos compañías concentraban más del $70 \%{ }^{3}$ del mercado aéreo de transporte de pasajeros en el país, el restante porcentaje, lo tenían 16 compañías con una participación poco sustancial, entre las que destacaban Taesa (10\%), Aerocalifornia (5\%), Saro (3\%) y Aviacsa (2\%).

Durante los 10 años que tardó la separación entre las dos compañías más importantes de México, la industria mostró señales de avance en materia de competencia. En 1995 se publicó la Ley de Aviación Civil, mediante la cual se estableció el marco legal de operación de las aerolíneas, asimismo, se promulgó la Ley de Aeropuertos con la que el gobierno inició el proceso de

\footnotetext{
${ }^{1}$ La Secretaría de Comunicaciones y Transportes (SCT) puso en marcha un nuevo "Esquema Rector del Sistema Nacional de Transporte Aéreo", con el que liberó a las aerolíneas troncales de la obligación de atender rutas que les resultaban poco rentables y otorgó mayor facilidad de ingreso a nuevos prestadores del servicio, especialmente la categoría nacional-regional y regional (alimentador).

${ }^{2}$ En diferentes ocasiones entre 1995 y 2000 la Cintra, pidió a la CFC reconsiderar su decisión sobre la venta individual de Aeroméxico y Mexicana. La CFC no alteró su decisión sobre el asunto, en parte debido a que los participantes en los inicios de los 90's no fueron tan exitosos como lo fueron al principio y la venta conjunta de ambas empresas tendría fuertes incentivos para incurrir en una conducta anticompetitiva (Ver CFC Expediente CNT-101-2007).

${ }^{3}$ Para una revisión de las participaciones anuales de mercado de ambas empresas ver la Tabla A.1 en el anexo.
} 
otorgamiento de concesiones para administrar, operar y explotar más de 30 aeropuertos del país, ${ }^{4}$ con el objetivo de hacer más eficiente la logística al interior de esas infraestructuras. Durante este proceso se crearon diversos Grupos Aeroportuarios ${ }^{5}$ que asumieron la administración de los distintos aeródromos del país, permaneciendo el Aeropuerto Internacional de México (AICM) bajo la responsabilidad del estado.

En 2005 se otorgaron nuevas concesiones a Aerolíneas de Bajo Costo (ABC's), entre las que se encontraban Volaris, Interjet, A Volar, Alma y Vivaaerobus; hasta ese momento el sector contaba con 11 competidores, 7 de ellos troncales y 4 regionales, la participación de Aeroméxico y Mexicana en el transporte doméstico de pasajeros se ubicó en un porcentaje cercano al 64\%, el nivel más bajo de cuota de mercado en los últimos años.

Para 2008, prácticamente más de la mitad del mercado del transporte aéreo de pasajeros estaba en poder de diferentes compañías distintas a Mexicana o Aeroméxico. Sin embargo, derivado de la crisis económica de 2009, la empresa Mexicana y diversas ABC's experimentaron dificultades financieras para continuar compitiendo dentro de la industria, ${ }^{6}$ por lo que a inicios de 2011 el número de actores económicos descendió a 7 participantes; esto favoreció a una nueva competencia por clientes y rutas que las empresas salientes dejaron de atender, traduciéndose en una redistribución del poder de mercado entre las líneas aéreas que permanecieron. De esta manera, entre los años de 2011 y 2013, emergió un nuevo escenario de la participación de Aeroméxico en el transporte de pasajeros domésticos que se ubicó en alrededor de $25 \%$.

\section{Marco regulatorio del sector}

El mercado de transporte aéreo en México se encuentra reglamentado principalmente por la Ley de Aviación Civil (LAC) publicada el 1995 y el Regla-

\footnotetext{
${ }^{4}$ Este cambio fue posible por la nueva Ley de Aeropuertos, expedida en 1995. Un elemento relevante durante el proceso de privatización de los aeropuertos es que la Secretaría de Hacienda y Crédito Público (SHCP) sería la encargada de determinar las tarifas máximas y las especificas en la prestación de servicios.

${ }^{5}$ El Grupo Aeroportuario Ciudad de México (GACM), Grupo Aeroportuario del Sureste (ASUR), Grupo Aeroportuario Centro-Norte (GACN) y Grupo Aeroportuario del Pacífico (GAP).

${ }^{6}$ La salida de empresas involucró tanto a aerolíneas nuevas (A Volar y Alma), como a tradicionales (Aerocalifornia, Azteca,Aviacsa y Mexicana), que no pudieron subsistir ante un entorno más adverso por causa de sus ineficiencias internas. Cardoso-Vargas (2015) documenta para el caso del sector externo mexicano que la crisis en México causó un efecto selección a favor de las firmas más productivas o con mayor capacidad para generar economías de escala.
} 
mento de la Ley de Aviación Civil (RLAC) dado a conocer en 1998. Estos ordenamientos tienen como propósito el regular la explotación, el uso o aprovechamiento del espacio aéreo situado sobre el territorio mexicano, respecto de la prestación y desarrollo de los servicios de transporte aéreo civil y los proporcionados por el estado. En la LAC se definen las reglas de operación de las diferentes actividades vinculadas al empleo de aeronaves comerciales, como las reglas para la obtención y revocación de las concesiones de transporte aéreo, el procedimiento de autorización de nuevas rutas en las cuales se brindará el servicio, el establecimiento de tarifas, entre otras.

Por su parte, la Ley de Aeropuertos (LDA) dada a conocer en 1995 y el Reglamento de la Ley de Aeropuertos (RLDA) del 2000, ${ }^{8}$ tienen como finalidad el regular la construcción, administración, operación y explotación de los aeródromos civiles, ${ }^{9}$ los cuales son parte integrante de las vías generales de comunicación, entre las que destaca la asignación de los slots ${ }^{10}$ de despegue y aterrizaje de las aeronaves comerciales y los criterios para la redistribución de esos slots en caso de que los aeropuertos se encuentren en una situación de saturación de sus operaciones.

En complemento de esas disposiciones la Ley Federal de Competencia Económica (LFCE) expedida en 2014 y el Reglamento de la Ley de Competencia Económica (RLCE) de 2007, establecen las reglas para que en los diferentes mercados, entre ellos el de transporte aéreo, se lleven a cabo prácticas de libre concurrencia y competencia entre los actores económicos involucrados; esto en armonía con el artículo 28 Constitucional. En cuanto a las actividades económicas que los extranjeros pueden realizar dentro del mercado de transporte nacional, la Ley de Inversión Extranjera (LIE) de 1993, regula las participaciones que pueden tener los inversionistas foráneos dentro de ese mercado.

\footnotetext{
${ }^{7}$ La LAC ha sido reformada o adicionada en cinco ocasiones (1998, 2001, 2006, 2013 y 2015) desde su publicación, en tanto que el RLAC cuenta con cuatro reformas con respecto a su versión original. ${ }^{8}$ La LDA cuenta con reformas en 2006, 2009 y 2015, por su parte, el RLAD en los años 2000 y 2003. ${ }^{9}$ Esta legislación abrió la posibilidad la participación de la inversión privada, incluyendo la de procedencia extranjera (artículo 19 LDA).

${ }^{10}$ El término refiere a las franjas horarias o itinerarios que se le establece a una aeronave para las actividades de aterrizaje o despegue dentro de un aeropuerto.
} 


\section{Condiciones de competencia}

\section{IV.I Concentración del mercado}

Un elemento fundamental para analizar el nivel de concentración del transporte aéreo de pasajeros, es cómo definir el "mercado relevante", es decir, el espacio geográfico en el que se ofrece o demanda el servicio o servicios similares. De acuerdo con la aplicación de los criterios plasmados en el Artículo 58 de la LFCE, se puede llegar a determinar que el transporte aéreo de pasajeros no cuenta con otros medios de transporte lo suficientemente cercanos, en precio y tiempos de recorrido, para ser considerados como posibles sustitutos. Asimismo, en virtud de que el vuelo entre dos ubicaciones normalmente no es sustituto de otro vuelo entre dos puntos distintos, se considera que el "mercado relevante" puede ser delimitado por su dimensión geográfica, que consiste en rutas origen-destino (OD) de punto a punto.

A pesar de contar con esta definición de "mercado relevante", el análisis se encuentra restringido por la falta de información oficial y pública respecto al comportamiento de los agentes económicos involucrados dentro de cada ruta OD. No obstante, a fin de solventar esta carencia y tener un panorama general sobre el nivel de concentración en el sector, los cálculos que se presentan en esta sección consideran los datos públicos de las compañías aéreas a nivel agregado respecto al transporte de pasajeros en servicio domésticos, que consideramos nuestro "mercado relevante".

Como medidas para determinar el grado de concentración en el mercado antes mencionado, se consideran los índices de concentración de Herfindahl (H) y de Dominancia (ID), de conformidad a lo dispuesto en la Resolución del 24 de julio de 1998 de la Comisión Federal de Competencia (RCFC 24/07/1998). ${ }^{11}$ Es así como para el cómputo de los índices de H y ID, se utilizó la información del número de pasajeros transportados por empresas nacionales en servicio doméstico regular, proveniente de la Secretaría de Comunicaciones y Transportes (SCT). ${ }^{12}$

\footnotetext{
${ }^{11}$ El uso de esta variable se encuentra en línea con lo dispuesto en el artículo primero de la RCFC 24/07/1998.

${ }^{12}$ El artículo segundo y tercero de este ordenamiento, establece que el Índice de Herfindahl (H) se calculará como la suma de los cuadrados de las participaciones de mercado de cada una de las compañías, es decir, $H=\sum_{i=1} n q_{i}^{2}$, dónde $q_{i}$ representa la contribución de la firma "i" dentro del mercado. Por su parte, el Índice de Dominancia (ID) se define como la suma de los cuadrados de las aportaciones al $\mathrm{H}$, lo que en términos algebraicos equivale a $I D=\sum_{i=1}{ }^{n} h_{i}^{2}$, donde $h_{i}=q_{i}^{2} / H \times 100$. Ambos índices son expresados en puntos y se encuentran acotados por arriba y por abajo, en el cual si toman un valor cercano a cero se consideran que existe competencia perfecta entre los actores
} 
En la gráfica 1, se observa que de 2000 a 2008, se presentó un proceso constante de disminución en la concentración del mercado de transporte de pasajeros en el mercado doméstico. ${ }^{13}$ En el año 2000 el índice H alcanzó un nivel de 3046 puntos, lo cual de acuerdo con el artículo cuarto de la RCFC 24/07/1998 ${ }^{14}$ apuntaba a que la concentración prevaleciente tenía posibilidades de afectar la competencia entre los agentes participantes en dicho mercado. En línea con este resultado el ID presentó para ese mismo año un valor de 4709 puntos, reflejando una mayor concentración además de una competencia desigual entre actores económicos de diferentes tamaños. En ambos casos, esta conclusión provenía por la presencia de las principales compañías aéreas hasta ese momento (Aeroméxico y Mexicana), las cuales concentraban el $76.5 \%$ del mercado.

Para 2008, el valor alcanzado por el índice $\mathrm{H}$ descendió para ubicarse en 1,370 puntos, lo que en términos de la RCFC 24/07/1998 apuntaba a que la concentración existente para ese año tenía pocas probabilidades de afectar el proceso de competencia y libre concurrencia dentro del mercado doméstico. Este resultado fue consecuencia, en gran medida por la pérdida paulatina de la proporción de mercado por parte de Aeroméxico y Mexicana, así como por la participación de más competidores, empresas de bajo costo, ${ }^{15}$ dentro del mercado local del país. Una evolución semejante también se percibió en el índice ID, no obstante, el valor alcanzado en 2008 indicó no sólo una menor concentración con respecto a los años previos, sino la presencia de diversos competidores de tamaño pequeño, con una cuota de mercado relativamente similar, entre algunos de ellos.

En contraste, para el período de 2009 al 2014 el índice H comenzó a registrar un crecimiento de manera anual derivado de una reconfiguración en las participaciones de las firmas prevalecientes dentro del mercado, ocasionada, entre otros factores, por: a) la salida de competidores como consecuencia de la

participantes dentro del mercado relevante; en contraparte, si el valor tiende a 10000 puntos se puede considerar que existe una alta concentración dentro del mercado similar a la de un monopolio. A diferencia del índice $\mathrm{H}$, el indicador ID toma en consideración el tamaño de los agentes económicos que participan dentro del mercado.

${ }^{13}$ Posterior al 2000, el nivel de concentración del índice $\mathrm{H}$ en el sector comenzó a disminuir año tras año, registrando una Tasa Media de Crecimiento Anual (TMCA) de $-10.7 \%$ durante el período de 2000 a 2008.

${ }^{14}$ Es importante aclarar que además del uso de estos indicadores, existen otros elementos que la actual Comisión Federal de Competencia Económica (Cofece) considera para evaluar si una concentración puede disminuir, dañar o impedir la competencia y libre concurrencia, como los mencionados en el artículo quinto de la RCFC 24/07/1998.

${ }^{15}$ Para una revisión de la evolución anual de la participación que tuvieron estas aerolíneas dentro del mercado ver la Tabla A.2 del anexo. 


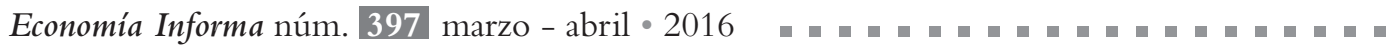

crisis económica de 2009, b) el cierre de operaciones por la compañía Mexicana en 2010 y c) la guerra de precios realizada entre compañías en 2013. El valor obtenido por el índice H para 2014 (1 974) representó un incremento nominal de 561 puntos con respecto a 2009 (1 413) y su valor fue muy cercano a la cifra observada en 2006 (1 973). ${ }^{16}$ En principio, esta evolución podría sugerir una tendencia a una nueva concentración del mercado, sin embargo, en términos del numeral 4.2 de la RCFC 24/07/1998 este comportamiento, aún no brinda indicios de que el proceso de competencia fue afectado. ${ }^{17}$ De igual manera, con la evidencia aportada por el índice ID no se puede considerar que la concentración prevaleciente hasta 2014 pueda impedir la competencia o la libre concurrencia dentro del mercado, pero su valor señala la presencia de agentes con una mayor diferencia en la cuota de mercado.

\section{Gráfica I}

Indices de concentración (H), (ID) y el número de competidores en el sector de transporte aéreo de pasajeros en México

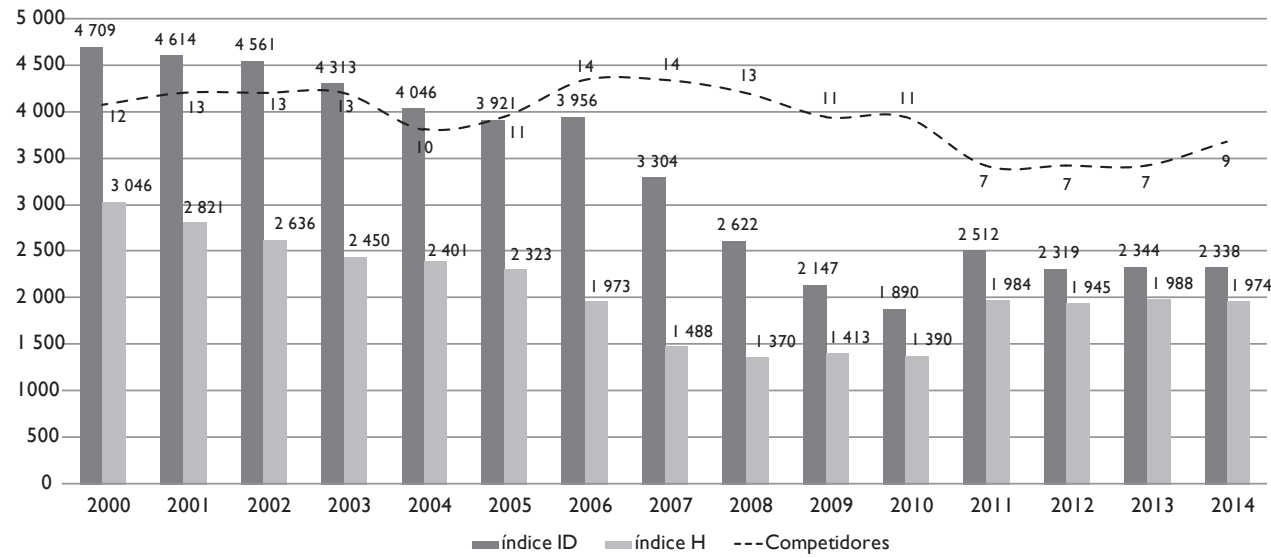

Fuente: elaboración propia con datos de la Dirección General de Aviación Civil de la sct, 2015. El índice $\mathrm{H}$ se refiere al cálculo el indicador de concetración de Herfindahl, por su parte el índice ID corresponde al índice de Dominancia.

\footnotetext{
${ }^{16}$ Las mayores variaciones del índice se presentaron durante el período posterior a la crisis de 2009 y la salida de la aerolínea Mexicana en 2010.

${ }^{17}$ Una revisión más puntual de los datos arroja que durante los años de 2010 y 2011, el índice H creció en 115 y 528 puntos, respectivamente; atendiendo lo dispuesto en el numeral 4.1 de la RCFC 24/07/1998 estos aumentos apuntaban a que el proceso de libre concurrencia dentro del sector se podría verse afectado, sin embargo, para los años posteriores el índice H ya no registró variaciones positivas anuales superiores a los 75 puntos.
} 


\section{IV.2 Precios}

En México, como en muchos países, no existe una base de datos que contenga información detallada de las tarifas aéreas a las que ofertan las distintas compañías a las diferentes rutas autorizadas. Para subsanar esta limitante en la realización de un análisis de precios, diversos investigadores como Ros (2010) y Boza y Oré (2007), llevaron a cabo una consulta permanente durante un largo tiempo de las tarifas publicadas por las diversas aerolíneas en sus páginas electrónicas, para las rutas que son sujetas de estudio. El presente documento también adolece de la misma falta de información, sin embargo, para realizar una evaluación del comportamiento de los precios se hace uso de los datos del Índice de Nacional de Precios Productos (INPP) para el transporte aéreo de pasajeros que publica el Instituto Nacional de Estadística y Geografía (INEGI).

El uso del INPP para el transporte aéreo cuenta con la ventaja de tener una alta representatividad, al contemplar a todos los actores involucrados dentro del sector, a pesar de que no distingue entre el transporte doméstico y el externo, se estima que su comportamiento capta en buena medida la evolución de los precios de las tarifas de las rutas locales.

En la gráfica 2 se presenta la variación interanual mensual del INPP conjuntamente con diferentes hechos relevantes acontecidos en el mercado de aerotransporte doméstico para un período de aproximadamente de 10 años. Durante los años de 2005 a 2007 el INPP del transporte aéreo presentó una desaceleración persistente en el crecimiento de los precios de dicho servicio, el cual coincide con el lapso en que el sector experimentó la menor concentración de mercado y que se relaciona con fenómenos que motivaron una mayor competencia, como la entrada de nuevos jugadores, entre ellos las ABC's, así como la venta a distintos particulares de las dos principales compañías, como Aeroméxico y Mexicana. 


\section{Gráfica 2}

Variación interanual mensual de INPP del transporte aéreo de pasajeros y eventos en el sector

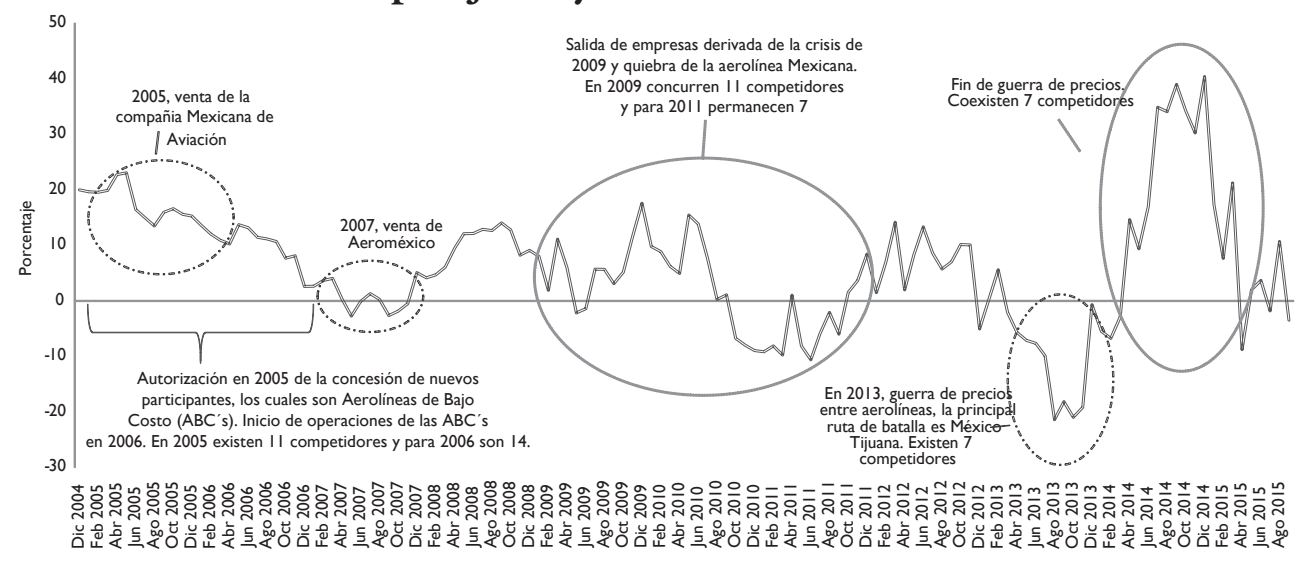

Fuente: Elaboración propia con datos del Indice Nacional de Precios al Productor (INPP) provenientes del INEGI y eventos acontecidos en el sector de transporte aéreo.

Posterior a la crisis económica de 2009 y a la quiebra de Mexicana en 2010, el INPP mostró signos de un decrecimiento en los precios del transporte aéreo de pasajeros durante distintos meses y que en junio de 2011 alcanzó una variación negativa interanual de $-10.6 \%$, cifra nunca antes vista, por lo menos, en los últimos cinco años. Esta evolución se explica, entre otros factores, por la competencia entre rutas y estrategias para captar un mayor número de clientes que terminó con una nueva repartición del mercado doméstico, prácticamente en 3 de los 7 jugadores que compartían hasta ese momento cuotas muy similares para el mercado local. En 2013 el INPP volvió a mostrar contracciones en su variación mensual a tasa anual, resultado de una guerra de precios entre las distintas compañías dentro del sector y que tuvo como finalidad allegarse de más pasajeros y contrarrestar la debilidad económica que inhibía los viajes aéreos.

Aunque las estrategias empleadas por los distintos actores en el mercado aéreo, fueron diferentes en las diversas rutas, ${ }^{18}$ de manera general todas las compañías realizaron una disminución de sus costos administrativos, a través de una baja en el personal contratado dedicado a esta actividad. ${ }^{19}$

\footnotetext{
${ }^{18}$ En la ruta México-Tijuana, principal bastión de batalla de las aerolíneas, el viaje sencillo con Interjet se cotizó en 2694 pesos, Aeroméxico 2532 pesos y Volaris en 2399 pesos. Nota periodística del diario El Financiero en julio de 2014.

${ }^{19}$ De acuerdo con cifras de la SCT, desde el 2010 las líneas aéreas troncales comenzaron a disminuir paulatinamente su planta laboral administrativa. Para 2013 el número de estos empleados fue el
} 


\section{Grafica 3}

Oferta de asientos

\section{Líneas aéreas nacionales troncales}

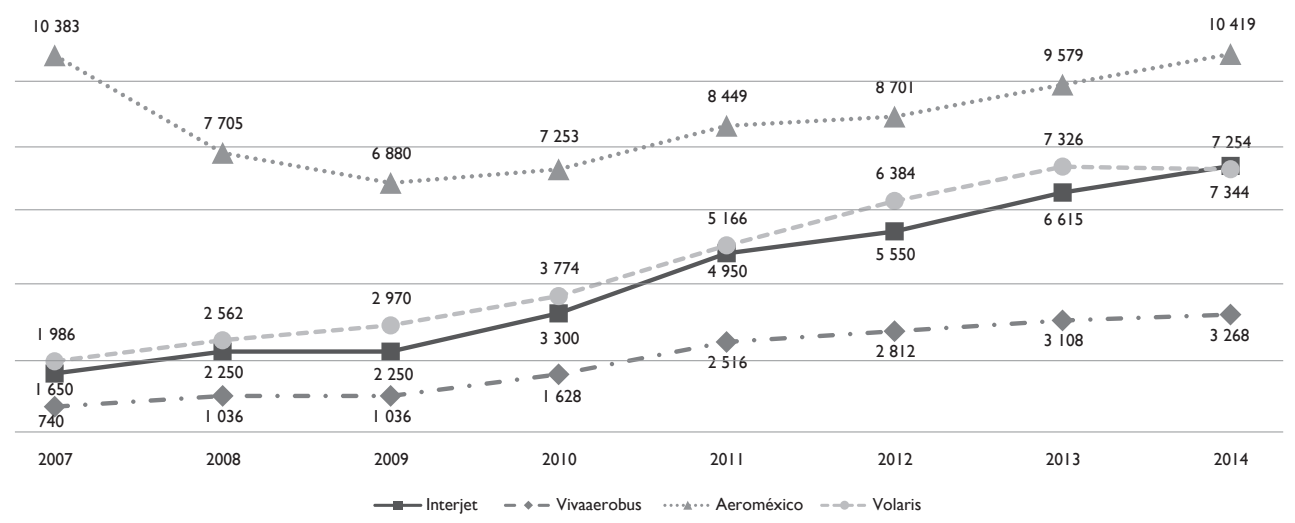

Fuente: elaboración propia con datos de la Dirección General de Aviación Civil de la sct, 2015.

Asimismo, las líneas áreas troncales y las regionales mostraron un comportamiento claramente diferenciado en cuanto a la oferta de asientos realizada; las primeras incrementaron la disponibilidad de lugares, en tanto que las segundas reflejaron una disminución en el número de asientos. Este comportamiento sugiere que la competencia por más clientes se llevo a cabo en rutas donde coexistían distintos actores, implementando una estrategia de economías de densidad, ${ }^{20}$ y no así en rutas regionales muy específicas donde sólo predominaba un sola compañía.

menor desde 1996, además este monto, representó menos de la mitad de empleos que se tenían registrados en 2010. En el caso de las aerolíneas regionales la contracción empezó desde 2007 y también en el 2013, se observó el menor número de empleados en ese rubro.

${ }^{20}$ Las economías de densidad podrán ser vistas como la disminución de los costes unitarios de una empresa aerocomercial, cuando la misma adiciona vuelos nuevos o más asientos en sus aeronaves, sin cambios en el factor de carga, distancia media o el número de aeropuertos servidos. (Caves, Christensen y Tretheway, 1984). 


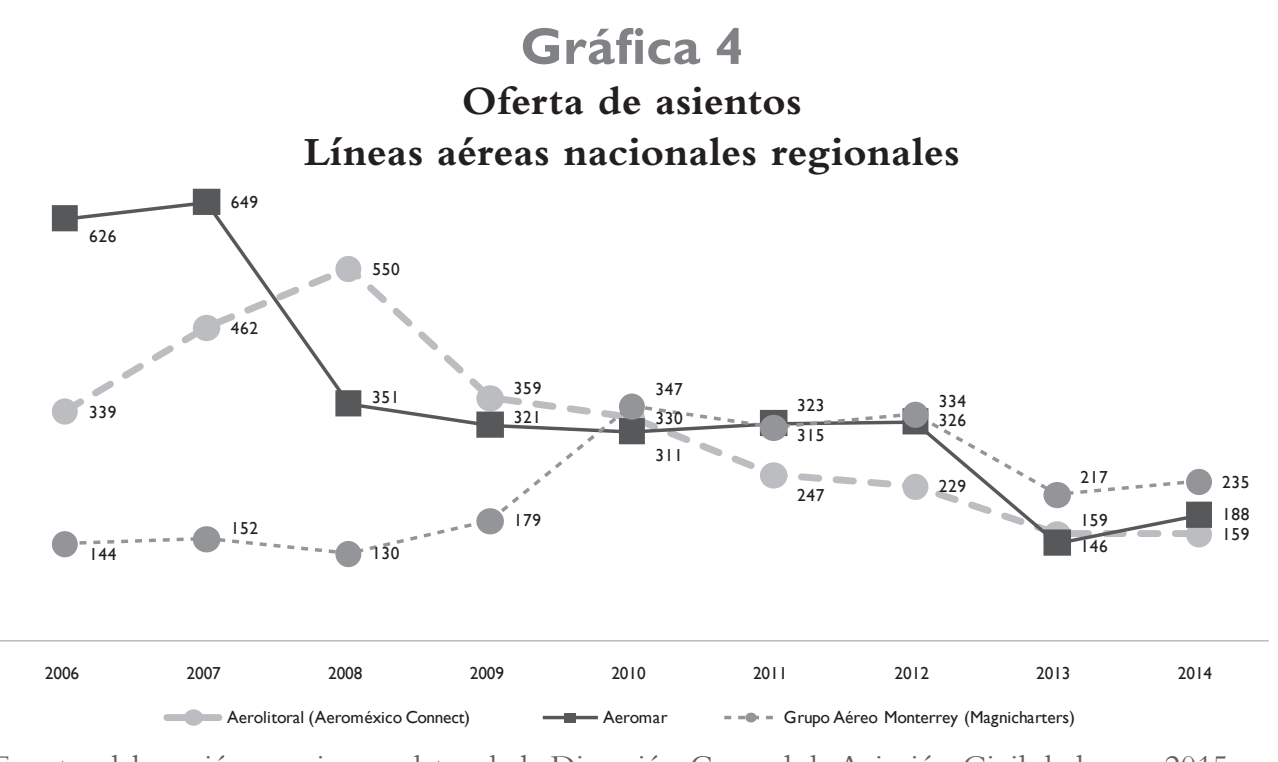

Fuente: elaboración propia con datos de la Dirección General de Aviación Civil de la SCT, 2015.

\section{IV.2.I Precios y poder de mercado}

Para examinar el grado en qué la competencia en el mercado nacional incidió en la variación de los precios en el sector, se elaboró un breve ejercicio estadístico mediante el uso de un modelo de regresión lineal simple. ${ }^{21}$

La estimación consiste en realizar una regresión de la variación mensual interanual del INPP con respecto índice de concentración ID, controlando los factores de oferta y demanda, así como shocks temporales que pudieron afectar la variación de precios. La racionalidad detrás de este ejercicio es evaluar la hipótesis de que los precios en el transporte aéreo guardan una estrecha relación con las condiciones de poder de mercado o concentración, en particular con el mercado doméstico; por lo que en caso de verificarse esta presunción, mostraría que en este sector existen condiciones para la fijación de precios.

En la columna 1 de la Tabla 1 se presentan los coeficientes obtenidos de la estimación de la variación mensual interanual del INPP con respecto al índice ID, los resultados muestran una relación positiva y estadísticamente significativa a $1 \%$, entre ambas variables. Esto brinda indicios respecto a la influencia que guarda la concentración económica del mercado doméstico en el crecimiento de los precios, es decir, cuanto mayor sea la cuota de mercado en pocas

\footnotetext{
${ }^{21}$ Es importante mencionar que los resultados obtenidos representan una aproximación básica en la evaluación y requieren de otros elementos para ser ampliados y validados.
} 
o una sola empresa, más grande será su capacidad para incrementar las tarifas de transporte de pasajeros. En particular, de existir una sola empresa que sirviera al mercado local, está tendría la capacidad de incrementar los precios en al menos un 46\%. Con el nivel de concentración observado hasta el 2014, las firmas domésticas establecidas tendrían posibilidad, en función de su interacción, de variar el precio de las tarifas en aproximadamente 8.8\%.

Aunque el resultado refleja una relación sólida, su significancia puede deberse a que esté capturando otros elementos relacionados con la determinación de precios, como las estrategias de oferta que ejercen los distintos jugadores dentro del sector, así como la demanda realizada por parte de los consumidores. Las estimaciones de las columnas (2) y (3) de la Tabla 1, muestran los coeficientes obtenidos después de controlar la oferta de asientos y el número de pasajeros transportados. En ambos casos el coeficiente del índice ID permanece positivo y altamente significativo.

\section{Tabla}

\section{Estimación de la variación de precios con respecto} a la concentración económica

\begin{tabular}{|l|r|r|r|r|}
\hline Variable dependiente: Variación & $(\boldsymbol{\Delta})$ mensual interanual INPP de transporte aéreo de pasajeros \\
\hline & $(1)$ & $(2)$ & $(3)$ & $(4)$ \\
\hline Índice ID & $0.00462^{\star}$ & $0.00428 \star \star$ & $0.00266^{\star \star}$ & $0.00506 \star \star$ \\
\hline & $(-4.58)$ & $(-4.63)$ & $(-2.87)$ & $(-3.78)$ \\
\hline Pasajeros transportados & & $-0.00131 \star \star$ & $-0.00185 \star \star$ & $-0.00284 \star \star$ \\
\hline & & $(-4.27)$ & $(-5.41)$ & $(-5.11)$ \\
\hline Oferta de asientos & & & $0.000861 \star \star$ & $0.00107 \star \star$ \\
\hline Constante & $-6.015 \star$ & $19.73 \star \star$ & $(-4.4)$ & $(-3.58)$ \\
\hline & $(-2.30)$ & $(-3.08)$ & $(-1.78)$ & $16.90+$ \\
\hline Dummy: años & No & No & No & Sí \\
\hline R2 & 0.114 & 0.259 & 0.374 & 0.659 \\
\hline AIC & 778.5 & 761.3 & 745 & 689.5 \\
\hline BIC & 783.9 & 769.4 & 755.7 & 713.6 \\
\hline F-statistics & 21.02 & 22.24 & 24.11 & 38.45 \\
\hline Observaciones & 108 & 108 & 108 & 108 \\
\hline
\end{tabular}

Fuente: estimaciones propias. Estadísticos $\mathrm{t}$ en paréntesis, los cuales son construidos usando errores estándar robustos. Las marcas $\star \star, \star y+$ indican un nivel de significancia de 1,5 y $10 \%$, respectivamente. 
A fin de tomar en cuenta shocks comunes que enfrentaron las compañías aéreas a través del tiempo, como la crisis de 2009, efectos de entradas y salidas de firmas en el sector o modificaciones en la política económica del país, en la columna (4) se incorporan efectos fijos anuales (variables dummy de año). Los parámetros estimados de todas las variables, aumentan su magnitud sin cambiar de signo o perder su significancia estadística, este incremento refleja la existencia de disparidades anuales en el poder de mercado y en la competencia que se presentó dentro del mercado doméstico de pasajeros y que incidió en el precio. Asimismo, los resultados alcanzados brindan soporte adicional a favor de la posible influencia que tiene la concentración económica en el mercado doméstico sobre los precios.

\section{IV.3 Competencia entre y dentro de aeropuertos}

El Aeropuerto Internacional de la Ciudad de México (AICM) históricamente ha tenido una participación destacada en el movimiento de pasajeros (nacionales e internacionales), de tal manera que por sí mismo en 2014 acumuló un $37.6 \%$ del tráfico total de pasajeros; lo que representa más del doble de la participación de su seguidor más cercano, Cancún, y cuatro veces la participación de Guadalajara, una de las ciudades más importantes del país

En cuanto al transporte de viajeros al interior del país, nuevamente el AICM resalta del resto de aeródromos a nivel nacional, concentrando en 2014 $35.7 \%$ de total de esos pasajeros y sólo por este lugar transitaron más individuos que los movilizados conjuntamente por los aeropuertos de Monterrey, Guadalajara, Cancún, Tijuana y Mérida. Estos datos reflejan la importancia que tiene el AICM en la competencia que realizan las empresas establecidas y las compañías que buscan ingresar a la industria (gráfica 5). 


\section{Gráfica 5 \\ Participación de mercado de los aeropuertos en transportación de pasajeros domésticos (2014)}

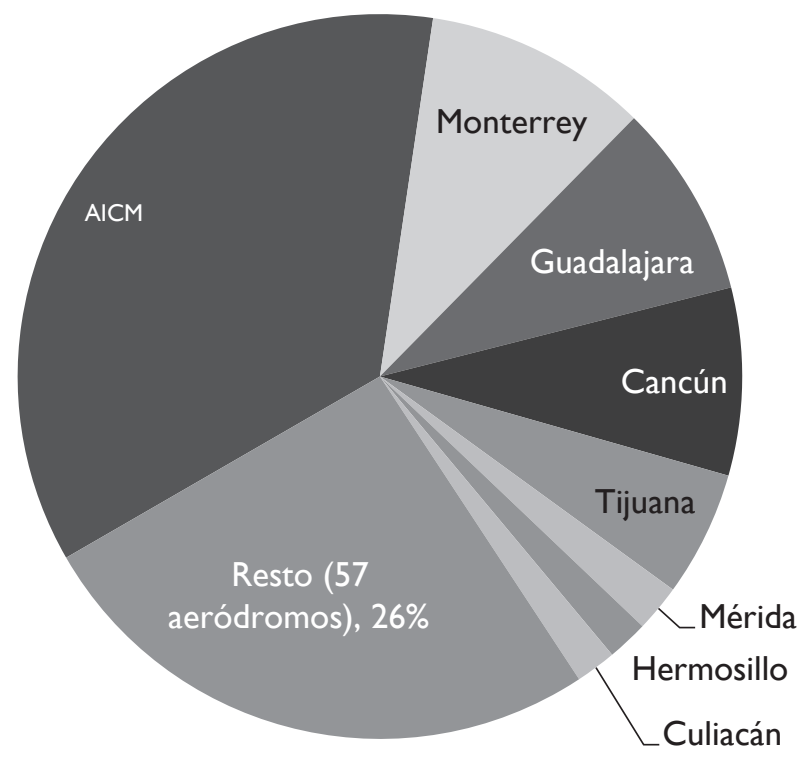

Fuente: elaboración propia con datos de la Dirección General de Aviación Civil de la sct, 2015.

Al comparar las participación del mercado nacional de acuerdo a los grupos que administran distintos aeródromos, se observa que después del AICM, el Grupo Aeroportuario del Pacifico (GAP) y el Grupo Aeroportuario del Sureste (ASUR) aglutinaron por separado una quinta parte de los usuarios que se transportaron dentro del país en 2014; en tanto, el Grupo Aeroportuario Centro Norte (GACN) concentró un 16\% de esos viajeros y Aeropuertos y Servicios Auxiliares (ASA) sólo alcanzó un 2.5\% (gráfica 6). En términos de competencia entre grupos, parece que la existencia de cuotas de mercado no tan disimiles entre tres de los concesionarios de los aeródromos y la aplicación de una metodología para la determinación de las tarifas de los servicios prestados por los aeropuertos, de conformidad con la LDA, apunta a un entorno propicio para la operación de las aerolíneas. Sin embargo, esta reflexión puede cambiar diametralmente al analizar las cuotas de mercado de las compañías aéreas al interior de los aeropuertos, como en el caso del AICM. 


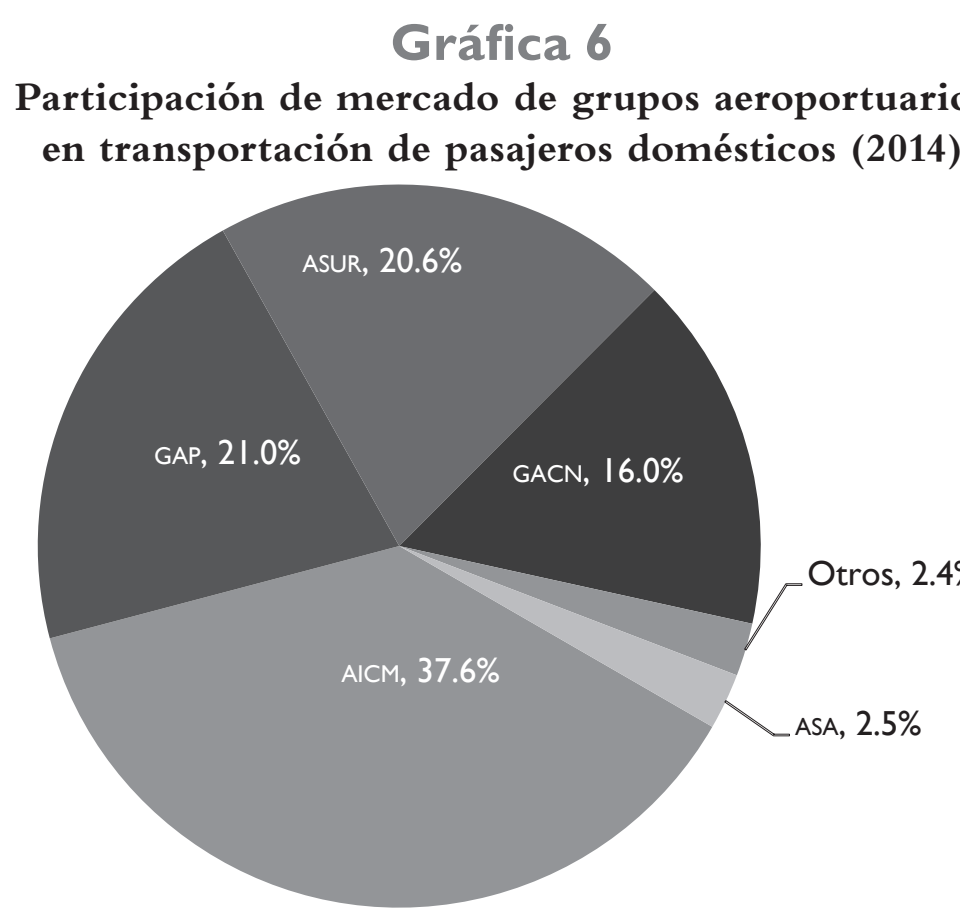

Fuente: elaboración propia con datos de la Dirección General de Aviación Civil de la SCT, 2015.

Desafortunadamente no existe información pública desglosada del tráfico de pasajeros que cada aerolínea realiza al interior de cada aeródromo, no obstante el análisis de López (2010) señala que en 2009 el 69.5\% de los slots de aterrizaje y despegue en el AICM eran utilizados por Mexicana (30.7\%) y Aeroméxico (29.1\%), el restante 30.5\% se encontraba repartido en las compañías Aeromar (9.7\%), Aviacsa (7.4\%), Interjet (6.3\%), entre otras. Este resultado da cuenta de una competencia desequilibrada entre los participantes al interior de esa infraestructura, además se percibió como un cuello de botella tanto para los competidores activos en ese momento como para los posibles entrantes.

\section{IV.4 Limitantes físicas y jurídicas para la competencia aérea}

A pesar de que en su momento el andamiaje legal implementado desde 1995, sirvió como un detonante para mejorar la competencia económica al interior de la industria de transporte aéreo, con el paso de los años han surgido algunas deficiencias en la aplicación operativa o restricciones impuestas en la normatividad vigente que derivan en menos competencia en la industria que a su vez se convierten en ineficiencias y en una pérdida del bienestar social. 
La asignación de los slots en los aeropuertos se realiza en función de las recomendaciones emitidas por un Comité ${ }^{22}$ de operación, en el cual participan representantes de las aerolíneas que brindan servicios en esas infraestructuras. Esta forma de distribuir los slots puede crear un ambiente de competencia inequitativo al interior de los aeropuertos, ya que las empresas entrantes, al no tener representatividad dentro del Comité, puede ser sujetas a que no les sean designadas franjas horarias para servir en una nueva ruta o reciban itinerarios en los que la demanda es baja o poco competitiva. Asimismo, en la repartición de slots se da preferencia a las aerolíneas con mayor antigüedad bajo un criterio de derecho adquirido ${ }^{23}$ (grandfatherclause), lo que puede propiciar que las compañías más antiguas soliciten la asignación de ciertos slots para impedir la entrada o crecimiento de otros jugadores. Este problema es aún más grave en aeropuertos con una alta saturación de flujos aéreos, como el AICM, donde además de limitar el ingreso de jugadores adicionales podría propiciar ineficiencias en el precio del servicio. ${ }^{24}$

En virtud de que la congestión del AICM llegó a niveles críticos, en 2013 la SCT emitió la Declaratoria de saturación para el aeropuerto, ${ }^{25}$ con lo cual se abrió la posibilidad de retirar los slots subutilizados ${ }^{26}$ y 1 levar a cabo una reasignación de los mismos de acuerdo a un criterio de subasta ${ }^{27}$ al mejor postor, sin embargo, a la fecha este procedimiento no se ha realizado. Otro problema asociado con los slots, tiene que ver con las tarifas que cobra el AICM, el cual por ser propiedad del gobierno federal no se encuentran sujetas al uso de la metodología utilizada por los aeropuertos privados, de conformidad con la LDA, con lo que las tarifas establecidas en el AICM no necesariamente incentivan

\footnotetext{
${ }^{22}$ El artículo 61 de la LDA establece que para la operación de los aeropuertos se constituirá un Comité conformado por el concesionario de dicha infraestructura, así como por los representantes de los concesionarios y permisionarios del servicio de transporte aéreo y de los prestadores de servicios, entre otros. Por su parte, el artículo 62 de ese mismo ordenamiento refiere a las recomendaciones que podrá realizar el Comité.

${ }^{23}$ El artículo 95 del RLA establece como uno de los criterios de asignación y priorización de los horarios un esquema basado en la antigüedad.

${ }^{24}$ Mediante la opinión PRES-10-096-2007-182, la Cofece se manifestó sobre las condiciones en las que operaba el AICM y emitió diversas recomendaciones enfocadas en promover una mayor competencia dentro del aeropuerto. Para 2010, nuevamente la Cofece se pronunció indicando que la forma de asignación de los slots es esencial para que las compañías alcancen una mayor eficiencia, por lo que realizó diversas recomendaciones sobre cómo mejorar el procedimiento vigente (PRES-10-096-2010-184).

${ }^{25}$ Publicada el 17 de abril de 2013 en el Diario Oficial de la Federación.

${ }^{26}$ El artículo 99 del RLA establece que el retiro de aquellos slots que: a) se utilizan menos del 85\% de las veces, una vez declarada la saturación: b) hubieren tenido demoras en un $15 \%$ o más; c) los horarios sean renunciados por los transportistas aéreos; y d) sean retirados cuando tengan adeudos vencidos en el pago de las contraprestaciones por los servicios aeroportuarios y a la navegación aérea.

${ }^{27}$ Artículos 95, 96 y 99 del RLA.
} 
una mayor competencia al interior de esa infraestructura, al ser un insumo que las aerolíneas utilizan para determinar sus precios.

Ros (2010) con el uso de datos de aproximadamente 500 rutas aéreas en México para el período de abril-agosto de 2009 y mediante la estimación de un modelo econométrico de cross-section, encuentra evidencia de que la alta saturación en el AICM tuvo un impacto negativo sobre las tarifas de los boletos de avión. El precio promedio de las rutas que utilizaban al AICM, como origen o destino, fue $33 \%$ superior a las rutas que no consideraban a ese aeropuerto dentro de su recorrido. Además, en dicho estudio se apunta que el precio promedio tiende a ser más bajo en aquellas rutas donde operan ABC's.

En virtud de que los problemas operativos y legales que enfrenta el AICM y de que es considerado el único Hub del país (López, 2010), estos no generan incentivos para que los agentes económicos lleven a cabo estrategias de servicio basadas en sistemas hub-and-spoke ${ }^{28}$ que les permitiría a las aerolíneas la formación de fuertes economías de densidad.

Otro aspecto importante es que en la actualidad existen disposiciones que restringen el incremento de nuevas rutas, en virtud de que cada adición representa una nueva autorización, además, el tiempo de aprobación en caso de ser largo podría retrasar la prestación del servicio y con ello no atender debidamente las demandas del mercado. Adicionalmente, una vez autorizadas las concesiones o las nuevas rutas, las compañías deben prestar el servicio dentro de un plazo máximo establecido, ya que en caso contrario dicho permiso será cancelado; ${ }^{29}$ esto obliga a las compañías a servir destinos por un tiempo definido, no obstante que existan razones financieras para no atender rutas por motivos de cambios repentinos en el mercado o estacionalidad en la demanda. Respecto al ingreso de capital extranjero en la industria, este se encuentra condicionado con una participación máxima de 25\% dentro de aerolíneas que prestan servicio doméstico dentro del país, lo que frena una mayor incorporación de compañías que ayudarían a dinamizar la competencia dentro del sector.

\footnotetext{
${ }^{28}$ Es un sistema de conexiones que permite reducir el número de rutas para comunicar los aeropuertos entre sí y consiste en concentrar el tráfico en determinados aeropuertos de gran capacidad o Hubs que se encargan de enlazar los de menor capacidad o importancia conocidos como Spokes.

${ }^{29}$ El artículo 19 de la LAC establece un plazo de 90 días para las nuevas rutas y el artículo 20 de esa misma Ley menciona un tiempo de 180 días, en el caso del otorgamiento de las concesiones.
} 


\section{Conclusiones y recomendaciones de política pública}

La evaluación de la competencia y libre entrada de nuevos jugadores en la industria del transporte aéreo en México, es compleja ya que involucra la participación de distintos agentes económicos cuyo marco de actuación es distinto. En primera instancia el análisis no simplemente involucra el conocimiento del marco regulatorio vigente, el ámbito económico y geográfico de competencia o los agentes económicos participantes, sino factores físicos, operativos o de infraestructura, que limitan o condicionan la estructura de costos de las compañías aéreas y su flexibilidad para operar. Esto en virtud de que condicionan su capacidad para generar economías de densidad o de red y a su vez limita la obtención de beneficios en precios y bienestar social que son ampliamente documentados por la literatura. Tales aspectos son revisados de manera sucinta en este trabajo, destacando aspectos relevantes y neurálgicos de la competencia en dicha industria.

Es de reconocer que un examen más amplio del sector, fuera del alcance de este documento, requiere del acceso a información más detallada, como el traslado de pasajeros y precios al nivel de aerolínea y ruta, toda vez que la publicada sólo permite evaluar al sector de manera agregada y representar sólo la punta del iceberg. ${ }^{30}$ No obstante, con la valoración realizada en este trabajo se puede obtener diversas conclusiones y recomendaciones de política pública.

En los últimos veinte años se observó la implementación de una política gubernamental enfocada a brindar condiciones de igualdad entre los agentes participantes en el sector, la entrada de nuevas compañías y el acceso a la administración de la infraestructura física al capital privado, lo que propició las condiciones para una competencia efectiva. En años recientes las cifras arrojan un contexto de competencia totalmente diferente al de inicios de los 90's, donde la mayor parte del poder de mercado del sector se concentraba en el duopolio conformado por Aeroméxico y; Mexicana en la actualidad el transporte aéreo de pasajeros se encuentra disputado por compañías tradicionales (como Aeroméxico) y distintas ABC's.

En la evolución reciente del sector es de destacar que derivado de la crisis económica de 2009 se presentó una redistribución del mercado nacional de pasajeros entre las líneas aéreas que si bien se tradujo en un incremento en la concentración económica del sector, no representa un aspecto preocupante en

\footnotetext{
${ }^{30}$ Por ejemplo, puede ser que la desregulación del sector brindó mayores condiciones para una mayor disputabilidad en ciertas rutas, derivando en menores tarifas; sin embargo, en otros casos pudo haber generado resultados contrarios (Valdés y Ramírez, 2011).
} 
términos de la RCFC 24/07/1998. Sin embargo, de acuerdo a las estimaciones presentadas en este documento permitió que las firmas establecidas tuvieran capacidad para incidir en el incremento de los precios en aproximadamente un $8 \%$.

Un aspecto de mayor relevancia, es el relacionado con las reglas de operación del AICM, en virtud de que el congestionamiento que registra se erige como una importante barrera a la competencia, tanto en la adición de nuevas rutas para las aerolíneas, como en las potenciales compañías que busquen operar en ese sitio. Las condiciones actuales propician que el precio de volar hacia o desde la Ciudad de México sea sustancialmente más caro que en otras rutas (Ros, 2010).

Ante la evidencia presentada en este documento, las recomendaciones para atender la problemática en el AICM parecen emanar de manera lógica y centrarse en: a) aplicar el mecanismo de subastas, previsto en la legislación actual; b) modificar la legislación para eliminar las cláusulas de "grandfather" y la operación de Comité; y c) modificar las reglas para la determinación de tarifas en el aeropuerto. Sin embargo, la primera sugerencia no parece ser del todo adecuada, ya que el implementar un mecanismo de subastas podría elevar el precio de los slots y por ende aumentar aún más el precio del transporte por parte de las aerolíneas, además de restar competitividad al AICM como principal Hub del país. En este sentido, el mecanismo de subastas debería estar complementado por otros criterios que limitarían el aumento desmedido de las tarifas, como el establecimiento de precios de congestión que han demostrado su efectividad en disminuir la saturación en grandes aeropuertos como los de Nueva York, Londres y Boston.

En cuanto a liberar la participación del capital extranjero en el mercado de transporte aéreo nacional a fin de atraer mayores recursos y experiencia, así como una mayor competencia, esta no parece ser una buena medida ya que podría tener un efecto contrario al esperado. En cambio, un incremento gradual $^{31}$ en la participación de dicho capital parece el camino más viable, de acuerdo a las experiencias registradas por otros sectores nacionales en el pasado.

\footnotetext{
${ }^{31}$ En un trabajo elaborado conjuntamente por la OECD-CFC (2012) se recomienda incrementar la participación del capital extranjero hasta un $49 \%$, basado en la experiencia exitosa que tuvo tal medida en los países de Brasil y Chile.
} 


\section{Bibliografía}

Boza, Sandy y Oré, Tilsa (2007), "El sector aerocomercial perunano: Situación y perespectivas". Gerencia de Estudios Económicos - INDECOPI. Documento de trabajo No 001 - 2007/GEE.

Cardoso-Vargas, Carlos-Enrique, (2015), "Productivity, Size and Exporting Dynamics of Firms: Evidence for Mexico". Available at SSRN: http://ssrn.com/ abstract=2639304 or http://dx.doi.org/10.2139/ssrn.2639304.

Caves, D., L. Christensen, y M. Tretheway (1984), "Economies of density versus economies of scale: why trunk and local servicies airline differ", Rand Journal of Economics, vol.15, n4, pp. 471-489.

Ley de Aviación Civil de 1995 y sus reformas, y su Reglamento con sus reformas. Ley de Aeropuertos de 1995 y sus reformas, y su Reglamento con sus reformas.

Ley de Federal de Competencia Económica de 2014.

López, Angel (2010), "Competencia en el sector de aerolíneas: La experiencia de México". Comisión Federal de Competencia, México.

OECD (2014). "Airline competition". Organisation for Economic Co-operation and Development. DAF/COMP/WD(2014) 47.

OECD (2014), "Eliminación de restricciones a la participación extranjera en México. Organisation for Economic Co-operation and Development y Comisión Federal de Competencia.

Oficio PRES-10-096-2007-182 de fecha $1^{\circ}$ de octubre de 2007. Disponible en en http://resoluciones.cfc.gob.mx/

Oficio PRES-10-096-2010-184 de fecha 3 de septiembre de 2010. Disponible en http://resoluciones.cfc.gob.mx/

Pérez, Eduardo (2010). "Regulación y competencia en el sector aéreo”. Comisión Federal de Competencia, México.

Resolución del 24 de julio de 1998 de la Comisión Federal de Competencia. (RCFC 24/07/1998).

Ros, Agustín (2010), “A Competition Policy Assessment of the Domestic Airline Sector in Mexico and Recommendations to Improve Competition", Documento preparado para el programa "Proceso para el fortalecimiento del marco regulatorio para la competitividad en México".

Valdés, Víctor y José Carlos, Ramírez, (2011), "Una evaluación sobre la desregulación del mercado de aerolíneas en México”. Economía Mexicana, Nueva Época. vol. Xx, núm. 1, pp. 5-35. 


\section{Anexo}

\section{Tabla A.I}

Evolución de la participación de Mexicana y Aeroméxico dentro del transporte de pasajeros domésticos (Cifras en porcentaje)

\begin{tabular}{|c|c|c|c|c|c|c|c|c|c|}
\hline Año & Aeroméxico & Mexicana & $\begin{array}{l}\text { Aeromé- } \\
\text { xico y } \\
\text { Mexicana }\end{array}$ & Resto & Año & Aeroméxico & Mexicana & $\begin{array}{c}\text { Aeromé- } \\
\text { xico y } \\
\text { Mexicana }\end{array}$ & Resto \\
\hline 1993 & 38.4 & 36.9 & 75.2 & 24.8 & 2004 & 33.8 & 31.6 & 65.4 & 34.6 \\
\hline 1994 & 37.5 & 33.1 & 70.6 & 29.4 & 2005 & 31.9 & 31.9 & 63.9 & 36.1 \\
\hline 1995 & 36.3 & 36.6 & 72.9 & 27.1 & 2006 & 28.2 & 30.7 & 58.9 & 41.1 \\
\hline 1996 & 37 & 35.6 & 72.6 & 27.4 & 2007 & 22.6 & 25.9 & 48.6 & 51.4 \\
\hline 1997 & 36.5 & 36.1 & 72.6 & 27.4 & 2008 & 19.6 & 23.6 & 43.2 & 56.8 \\
\hline 1998 & 35.1 & 33.7 & 68.9 & 31.1 & 2009 & 18.6 & 21.9 & 40.5 & 59.5 \\
\hline 1999 & 36.1 & 31.8 & 67.9 & 32.1 & 2010 & 20.7 & 14.6 & 35.3 & 64.7 \\
\hline 2000 & 40.3 & 36.2 & 76.5 & 23.5 & 2011 & 27.1 & 0 & 27.1 & 72.9 \\
\hline 2001 & 38.4 & 34.9 & 73.4 & 26.6 & 2012 & 24.5 & 0 & 24.5 & 75.5 \\
\hline 2002 & 37.7 & 32.8 & 70.4 & 29.6 & 2013 & 23.3 & 0 & 23.3 & 76.7 \\
\hline 2003 & 34.7 & 32.6 & 67.3 & 32.7 & 2014 & 23.4 & 0 & 23.4 & 76.6 \\
\hline
\end{tabular}

Fuente: elaboración propia con datos de la Dirección General de Aviación Civil de la sct, 2015.

\section{Tabla A.2}

Evolución de la participación de las Aerolíneas de Bajo Costo (ABC's) dentro del transporte de pasajeros domésticos (Cifras en porcentaje)

\begin{tabular}{|l|r|r|r|r|r|r|r|r|r|}
\hline & 2006 & 2007 & 2008 & 2009 & 2010 & 2011 & 2012 & 2013 & 2014 \\
\hline Interjet & 4.32 & 5.64 & 8.6 & 9.94 & 13.06 & 21.21 & 21.34 & 22.48 & 21.36 \\
\hline VivaAerobus & 0.23 & 3.54 & 3.99 & 4.65 & 7.24 & 9.96 & 10.75 & 10.16 & 9.76 \\
\hline Volaris & 3.09 & 6.34 & 9.59 & 10.66 & 13.7 & 18.67 & 20.73 & 22.72 & 23.01 \\
\hline Alma & 0.72 & 2.57 & 2.46 & - & - & - & - & - & - \\
\hline A Volar & 1.48 & 2.77 & 1.41 & - & - & - & - & - & - \\
\hline Total & 9.84 & 20.85 & 26.06 & 25.25 & 34.01 & 49.84 & 52.81 & 55.36 & 54.13 \\
\hline Mexica y Aeroméxico & 58.91 & 48.57 & 43.24 & 40.51 & 35.28 & 27.08 & 24.5 & 23.34 & 23.37 \\
\hline Otras compañías aéreas & 31.25 & 30.58 & 30.71 & 34.24 & 30.71 & 23.08 & 22.69 & 21.29 & 22.5 \\
\hline
\end{tabular}

Fuente: elaboración propia con datos de la Dirección General de Aviación Civil de la sCT, 2015. 